\title{
Blow-Up Analysis for a Quasilinear Parabolic Equation with Inner Absorption and Nonlinear Neumann Boundary Condition
}

\author{
Zhong Bo Fang and Yan Chai \\ School of Mathematical Sciences, Ocean University of China, Qingdao 266100, China \\ Correspondence should be addressed to Zhong Bo Fang; fangzb7777@hotmail.com
}

Received 24 February 2014; Revised 11 April 2014; Accepted 11 April 2014; Published 30 April 2014

Academic Editor: Zhi-Bo Huang

Copyright ( 2014 Z. B. Fang and Y. Chai. This is an open access article distributed under the Creative Commons Attribution License, which permits unrestricted use, distribution, and reproduction in any medium, provided the original work is properly cited.

We investigate an initial-boundary value problem for a quasilinear parabolic equation with inner absorption and nonlinear Neumann boundary condition. We establish, respectively, the conditions on nonlinearity to guarantee that $u(x, t)$ exists globally or blows up at some finite time $t^{*}$. Moreover, an upper bound for $t^{*}$ is derived. Under somewhat more restrictive conditions, a lower bound for $t^{*}$ is also obtained.

\section{Introduction}

We are concerned with the global existence and blow-up phenomenon for a quasilinear parabolic equation with nonlinear inner absorption term

$$
u_{t}=\left[\left(|\nabla u|^{p}+1\right) u_{, i}\right]_{, i}-f(u), \quad(x, t) \in \Omega \times\left(0, t^{*}\right),
$$

subjected to the nonlinear Neumann boundary and initial conditions

$$
\begin{gathered}
\left(|\nabla u|^{p}+1\right) \frac{\partial u}{\partial \nu}=g(u), \quad(x, t) \in \partial \Omega \times\left(0, t^{*}\right), \\
u(x, 0)=u_{0}(0) \geq 0, \quad x \in \bar{\Omega},
\end{gathered}
$$

where $\Omega$ is a bounded star-shaped region of $R^{N}(N \geq 2)$ with smooth boundary $\partial \Omega, v$ is the unit outward normal vector on $\partial \Omega, p \geq 0, t^{*}$ is the blow-up time if blow-up occurs, or else $t^{*}=+\infty$, the symbol, $i$ denotes partial differentiation with respect to $x_{i}, i=1,2, \ldots, N$, the repeated index indicates summation over the index, and $\nabla$ is gradient operator.

Many physical phenomena and biological species theories, such as the concentration of diffusion of some nonNewton fluid through porous medium, the density of some biological species, and heat conduction phenomena, have been formulated as parabolic equation (1) (see [1-3]). The nonlinear Neumann boundary condition (2) can be physically interpreted as the nonlinear radial law (see $[4,5])$.
In the past decades, there have been many works dealing with existence and nonexistence of global solutions, blow-up of solutions, bounds of blow-up time, blow-up rates, blowup sets, and asymptotic behavior of solutions to nonlinear parabolic equations; see the books [6-8] and the survey papers [9-11]. Specially, we would like to know whether the solution blows up and at which time when blow-up occurs. A variety of methods have been used to study the problem above (see [12]), and in many cases, these methods, used to show that solutions blow up, often provide an upper bound for the blow-up time. However, lower bounds for blow-up time may be harder to be determined. For the study of the initial boundary value problem of a parabolic equation with homogeneous Dirichlet boundary condition, see [13, 14]. Payne et al. [13] considered the following quasilinear parabolic equation:

$$
u_{t}=\operatorname{div}\left(\rho|\nabla u|^{2} \nabla u\right)+f(u), \quad(x, t) \in \Omega \times\left(0, t^{*}\right),
$$

where $\Omega$ is a bounded domain in $R^{3}$ with smooth boundary $\partial \Omega$. To get the lower bound for the blow-up time, the authors assumed that $\rho$ is a positive $C^{1}$ function which satisfies

$$
\rho(s)+s \rho^{\prime}(s)>0, \quad s>0 .
$$

The lower bound for the blow-up time of solution to (4) with Robin boundary condition was obtained in [15], where $\rho$ is 
also assumed to satisfy the condition (5). However, under this boundary condition, the best constant of Sobolev inequality used in [13] is no longer applicable. They imposed suitable conditions on $f$ and $\rho$ and determined a lower bound for the blow-up time if blow-up occurs and determined when blow-up cannot occur. Marras and Vernier Piro [14] studied the nonlinear parabolic problem with time dependent coefficients

$$
\begin{array}{r}
k_{1}(t) \operatorname{div}\left(g\left(|\nabla u|^{2} \nabla u\right)\right)+k_{2}(t) f(u)=k_{3}(t) u_{t}, \\
(x, t) \in \Omega \times\left(0, t^{*}\right),
\end{array}
$$

where $\Omega$ is a bounded domain in $R^{N}$ with smooth boundary $\partial \Omega$. Under some conditions on the data and geometry of the spatial domain, they obtained upper and lower bounds of the blow-up time. Moreover, the sufficient conditions for global existence of the solution were derived.

For the study of the initial boundary value problem of a parabolic equation with Robin boundary condition, we refer to [15-19]. Li et al. [16] investigated the problem of the nonlinear parabolic equation

$$
u_{t}=\left[\left(|\nabla u|^{p}+1\right) u_{, i}\right]_{, i}+f(u), \quad(x, t) \in \Omega \times\left(0, t^{*}\right),
$$

where $\Omega$ is a bounded domain in $R^{3}$ with smooth boundary $\partial \Omega$. They derived the lower bound for the blow-up time when the blow-up occurs. Clearly, $|\nabla u|^{p}+1$ does not satisfy the condition (5). Enache [17] discussed the quasilinear parabolic problem

$$
u_{t}=\left(g(u) u_{, i}\right)_{, i}+f(u)
$$

where $\Omega$ is a bounded domain in $R^{N}(N \geq 2)$ with smooth boundary $\partial \Omega$. By virtue of a first-order differential inequality technique, they showed the sufficient conditions to guarantee that the solution $u(x, t)$ exists globally or blows up. In addition, a lower bound for the blow-up time when blowup occurs was also obtained. Ding [18] studied the nonlinear parabolic problem

$$
(b(u))_{t}=\nabla \cdot(g(u) \nabla u)+f(u), \quad(x, t) \in \Omega \times\left(0, t^{*}\right),
$$

where $\Omega$ is a bounded domain in $R^{3}$ with smooth boundary $\partial \Omega$. They derived conditions on the data which guarantee the blow-up or the global existence of the solution. A lower bound on blow-up time when blow-up occurs was also obtained. For the problem of the nonlinear nonlocal porous medium equation, we read the paper of Liu [19].

Recently, for the problems with nonlinear Neumann boundary conditions, Payne et al. [20] studied the semilinear heat equation with inner absorption term

$$
u_{t}=\Delta u-f(u), \quad(x, t) \in \Omega \times\left(0, t^{*}\right) .
$$

They established conditions on nonlinearity to guarantee that the solution $u(x, t)$ exists for all time $t>0$ or blows up at some finite time $t^{*}$. Moreover, an upper bound for $t^{*}$ was derived. Under somewhat more restrictive conditions, a lower bound for $t^{*}$ was derived. Thereafter, they considered the quasilinear parabolic equation

$$
u_{t}=\nabla \cdot\left(|\nabla u|^{p} \nabla u\right), \quad(x, t) \in \Omega \times\left(0, t^{*}\right),
$$

and they showed that blow-up occurs at some finite time under certain conditions on the nonlinearities and the data; upper and lower bounds for the blow-up time were derived when blow-up occurs; see [21]. Liu et al. The authors [22, 23] studied the reaction diffusion problem with nonlocal source and inner absorption terms or with local source and gradient absorption terms. Very recently, Fang et al. [24] considered lower bounds estimate for the blow-up time to nonlocal problem with homogeneous Dirichlet or Neumann boundary condition.

Motivated by the above work, we intend to study the global existence and the blow-up phenomena of problem (1)(3), and the results of the semilinear equations are extended to the quasilinear equations. Unfortunately, the techniques used for semilinear equation to analysis of blow-up phenomena are no longer applicable to our problem. As a consequence, by using the suitable techniques of differential inequalities, we establish, respectively, the conditions on the nonlinearities $f$ and $g$ to guarantee that $u(x, t)$ exists globally or blows up at some finite time. If blow-up occurs, we derive upper and lower bounds of the blow-up time.

The rest of our paper is organized as follows. In Section 2, we establish conditions on the nonlinearities to guarantee that $u(x, t)$ exists globally. In Section 3, we show the conditions on data forcing the solution $u(x, t)$ to blow up at some finite time $t^{*}$ and obtain an upper bound for $t^{*}$. A lower bound of blow-up time under some assumptions is derived in Section 4.

\section{The Global Existence}

In this section, we establish the conditions on the nonlinearity $f$ and nonlinearity $g$ to guarantee that $u(x, t)$ exists globally. We state our result as follows.

Theorem 1. Assume that the nonnegative functions $f$ and $g$ satisfy

$$
\begin{aligned}
& f(\xi) \geq k_{1} \xi^{q}, \quad \xi \geq 0, \\
& g(\xi) \leq k_{2} \xi^{s}, \quad \xi \geq 0,
\end{aligned}
$$

where $k_{1}>0, k_{2} \geq 0, s>1,2 s<q+1$, and $s-1<p<q-1$. Then the (nonnegative) solution $u(x, t)$ of problem (1)-(3) does not blow up; that is, $u(x, t)$ exists for all time $t>0$.

Proof. Set

$$
\Psi(t)=\int_{\Omega} u^{2} d x
$$


Similar to Theorem 2.1 in [20], we get

$$
\begin{aligned}
\Psi^{\prime}(t) \leq & \left\{2 \delta^{2} \int_{\Omega} u^{2 s} d x-k_{1} \int_{\Omega} u^{q+1} d x\right\} \\
& +\left\{\frac{2 k_{2} N}{\rho_{0}} \int_{\Omega} u^{s+1} d x\right. \\
& \left.-2 \int_{\Omega}|\nabla u|^{p+2} d x-k_{1} \int_{\Omega} u^{q+1} d x\right\} \\
= & I_{1}+I_{2},
\end{aligned}
$$

where $\delta=k_{2}(s+1) d / 2 \rho_{0}, \rho_{0}=\min _{x \in \partial \Omega}(x \cdot v), d=$ $\max _{x \in \partial \Omega}|x|$, and

$$
\begin{aligned}
I_{1} \leq & \left(\int_{\Omega} u^{q+1} d x\right)^{(s+1) /(q+1)} \\
& \times\left\{\widetilde{A_{1}}|\Omega|^{(q-s) /(q+1)}-\widetilde{A_{2}}\left(\int_{\Omega} u^{q+1} d x\right)^{(q-s) /(q+1)}\right\},
\end{aligned}
$$

where $\widetilde{A_{1}}=2 \delta^{2} \alpha \varepsilon^{(\alpha-1) / \alpha}, \widetilde{A_{2}}=k_{1}-2 \delta^{2}(1-\alpha) \varepsilon, \alpha=(q+1-$ $2 s) /(q-s)<1, \varepsilon>0$.

Next, we estimate $I_{2}=\left(2 k_{2} N / \rho_{0}\right) \int_{\Omega} u^{s+1} d x-2 \int_{\Omega}|\nabla u|^{p+2}$ $d x-k_{1} \int_{\Omega} u^{q+1} d x$. Since

$$
\left|\nabla u^{(p / 2)+1}\right|^{2}=\left(\frac{p}{2}+1\right)^{2} u^{p}|\nabla u|^{2}
$$

it follows from Hölder inequality that

$$
\begin{aligned}
\int_{\Omega}\left|\nabla u^{(p / 2)+1}\right|^{2} d x \leq & \left(\frac{p}{2}+1\right)^{2}\left(\int_{\Omega}|\nabla u|^{p+2} d x\right)^{2 /(p+2)} \\
& \times\left(\int_{\Omega} u^{p+2} d x\right)^{p /(p+2)} \cdot
\end{aligned}
$$

Furthermore, we have

$$
\int_{\Omega} u^{p+2} d x \leq\left[\frac{(p+2)^{2}}{4 \lambda_{1}}\right]^{(p / 2)+1} \int_{\Omega}|\nabla u|^{p+2} d x
$$

which follows from (17) and membrane inequality

$$
\lambda_{1} \int_{\Omega} \omega^{2} d x \leq \int_{\Omega}|\nabla \omega|^{2} d x
$$

where $\lambda_{1}$ is the first eigenvalue in the fixed membrane problem

$$
\Delta \omega+\lambda \omega=0, \quad \omega>0 \text { in } \Omega, \omega=0 \text { on } \partial \Omega .
$$

Combining $I_{2}$ and (18), we have

$$
\begin{aligned}
I_{2} \leq & \frac{2 k_{2} N}{\rho_{0}} \int_{\Omega} u^{s+1} d x-2\left[\frac{4 \lambda_{1}}{(p+2)^{2}}\right]^{(p / 2)+1} \\
& \times \int_{\Omega} u^{p+2} d x-k_{1} \int_{\Omega} u^{q+1} d x . \\
= & \left\{\frac{2 k_{2} N}{\rho_{0}} \int_{\Omega} u^{s+1} d x-3\left[\frac{4 \lambda_{1}}{(p+2)^{2}}\right]^{(p / 2)+1} \int_{\Omega} u^{p+2} d x\right\} \\
& +\left\{\left[\frac{4 \lambda_{1}}{(p+2)^{2}}\right]^{(p / 2)+1} \int_{\Omega} u^{p+2} d x-k_{1} \int_{\Omega} u^{q+1} d x\right\} \\
= & I_{21}+I_{22} .
\end{aligned}
$$

Making use of Hölder inequality, we obtain

$$
\begin{aligned}
& \int_{\Omega} u^{s+1} d x \leq\left(\int_{\Omega} u^{p+2} d x\right)^{(s+1) /(p+2)}|\Omega|^{(p-s+1) /(p+2)} \\
& \Psi(t)=\int_{\Omega} u^{2} d x \leq\left(\int_{\Omega} u^{s+1} d x\right)^{2 /(s+1)}|\Omega|^{(s-1) /(s+1)} .
\end{aligned}
$$

Combining (21), (22) with (23), we get

$$
I_{21} \leq\left(\int_{\Omega} u^{s+1} d x\right)\left\{B_{1}-B_{2} \Psi^{(p-s+1) / 2}\right\},
$$

with

$$
B_{1}=\frac{2 k_{2} N}{\rho_{0}}, \quad B_{2}=3\left[\frac{4 \lambda_{1}}{(p+2)^{2}}\right]^{(p / 2)+1}|\Omega|^{-(p-s+1) / 2} \text {. }
$$

Applying Hölder inequality, we obtain

$$
\begin{aligned}
& \int_{\Omega} u^{p+2} d x \leq\left(\int_{\Omega} u^{q+1} d x\right)^{(p+2) /(q+1)}|\Omega|^{(q-p-1) /(q+1)}, \\
& \Psi(t)=\int_{\Omega} u^{2} d x \leq\left(\int_{\Omega} u^{q+1} d x\right)^{2 /(q+1)}|\Omega|^{(q-1) /(q+1)} .
\end{aligned}
$$

It follows from (26) that

$$
I_{22} \leq\left(\int_{\Omega} u^{q+1} d x\right)^{(p+2) /(q+1)}\left\{C_{1}-C_{2} \Psi^{(q-p-1) / 2}\right\},
$$

where

$$
\begin{aligned}
& C_{1}=\left[\frac{4 \lambda_{1}}{(p+2)^{2}}\right]^{(p / 2)+1}|\Omega|^{(q-p-1) /(q+1)}, \\
& C_{2}=k_{1}|\Omega|^{(1-q)(q-p-1) / 2(q+1)} .
\end{aligned}
$$


Combining (14), (15), (21), and (24) with (27), we obtain

$$
\begin{aligned}
\Psi^{\prime}(t) \leq & \left(\int_{\Omega} u^{q+1} d x\right)^{(s+1) /(q+1)}\left\{A_{1}-A_{2} \Psi(t)^{(q-s) / 2}\right\} \\
& +\left(\int_{\Omega} u^{s+1} d x\right)\left\{B_{1}-B_{2} \Psi^{(p-s+1) / 2}\right\} \\
& +\left(\int_{\Omega} u^{q+1} d x\right)^{(p+2) /(q+1)}\left\{C_{1}-C_{2} \Psi^{(q-p-1) / 2}\right\},
\end{aligned}
$$

with

$$
A_{1}=\widetilde{A_{1}}|\Omega|^{(q-s) /(q+1)}, \quad A_{2}=\widetilde{A_{2}}|\Omega|^{(1-q)(q-s) / 2(q+1)} .
$$

We conclude from (29) that $\Psi(t)$ is decreasing in each time interval on which we obtain

$$
\Psi(t) \geq \max \left\{\left(\frac{A_{1}}{A_{2}}\right)^{2 /(q-s)},\left(\frac{B_{1}}{B_{2}}\right)^{2 /(p-s+1)},\left(\frac{C_{1}}{C_{2}}\right)^{2 /(q-p-1)}\right\},
$$

so that $\Psi(t)$ remains bounded for all time under the conditions in Theorem 1. This completes the proof of Theorem 1.

\section{Blow-Up and Upper Bound of $t^{*}$}

In this section, $\Omega$ needs not to be star-shaped. We establish the conditions to assure that the solution of (1)-(3) blows up at finite time $t^{*}$ and derive an upper bound for $t^{*}$. More precisely we establish the following result.

Theorem 2. Let $u(x, t)$ be the classical solution of problem (1)(3). Assume that the nonnegative and integrable functions $f$ and $g$ satisfy

$$
\begin{array}{ll}
\xi f(\xi) \leq 2(1+\alpha) F(\xi), & \xi \geq 0, \\
\xi g(\xi) \geq 2(1+\beta) G(\xi), & \xi \geq 0,
\end{array}
$$

with

$$
F(\xi)=\int_{0}^{\xi} f(\eta) d \eta, \quad G(\xi)=\int_{0}^{\xi} g(\eta) d \eta
$$

where $\alpha \geq 0$,

$$
\beta \geq \max \left(\frac{p}{2}, \alpha\right) .
$$

Moreover assume that $\Phi(0) \geq 0$ with

$$
\begin{aligned}
\Phi(t)= & 2 \int_{\partial \Omega} G(u) d S-\int_{\Omega}|\nabla u|^{2}\left(1+\frac{2}{p+2}|\nabla u|^{p}\right) d x \\
& -2 \int_{\Omega} F(u) d x .
\end{aligned}
$$

Then the solution $u(x, t)$ of problem (1)-(3) blows up at some finite time $t^{*}<T$ with

$$
T=\frac{\Psi(0)}{2 \beta(1+\beta) \Phi(0)}, \quad \beta>0,
$$

where $\Psi(t)$ is defined in (13). If $\beta=0$, we have $T=\infty$.
Proof. We compute

$$
\begin{aligned}
\Psi^{\prime}(t)= & 2 \int_{\Omega} u u_{t} d x=2 \int_{\Omega} u\left[\left(\left(|\nabla u|^{p}+1\right) u_{, i}\right)_{, i}-f(u)\right] d x \\
= & 2 \int_{\partial \Omega} u\left(|\nabla u|^{p}+1\right) \frac{\partial u}{\partial \nu} d S-2 \int_{\Omega}\left(|\nabla u|^{p}+1\right)|\nabla u|^{2} d x \\
& -2 \int_{\Omega} u f(u) d x \\
= & 2 \int_{\partial \Omega} u g(u) d S-2 \int_{\Omega}\left(|\nabla u|^{p}+1\right)|\nabla u|^{2} d x \\
& -2 \int_{\Omega} u f(u) d x .
\end{aligned}
$$

Making use of the hypotheses stated in Theorem 2, we have

$$
\Psi^{\prime}(t) \geq 2(1+\beta) \Phi(t) .
$$

Differentiating (35), we derive

$$
\begin{aligned}
\Phi^{\prime}(t)= & 2 \int_{\partial \Omega} g(u) u_{t} d S-\int_{\Omega}\left(|\nabla u|^{p}+1\right)\left(|\nabla u|^{2}\right)_{t} d x \\
& -2 \int_{\Omega} f(u) u_{t} d x .
\end{aligned}
$$

Integrating the identity $\nabla \cdot\left(u_{t}\left(|\nabla u|^{p}+1\right) \nabla u\right)=u_{t} \nabla \cdot\left(\left(|\nabla u|^{p}+\right.\right.$ $1) \nabla u)+(1 / 2)\left(|\nabla u|^{p}+1\right)\left(|\nabla u|^{2}\right)_{t}$ over $\Omega$, we get

$$
\begin{aligned}
\int_{\Omega} & \left(|\nabla u|^{p}+1\right)\left(|\nabla u|^{2}\right)_{t} d x \\
= & 2 \int_{\Omega} \nabla \cdot\left(u_{t}\left(|\nabla u|^{p}+1\right) \nabla u\right) d x \\
& -2 \int_{\Omega} u_{t} \nabla \cdot\left(\left(|\nabla u|^{p}+1\right) \nabla u\right) d x \\
= & 2 \int_{\partial \Omega} u_{t}\left(|\nabla u|^{p}+1\right) \nabla u \cdot v d S \\
& -2 \int_{\Omega} u_{t} \nabla \cdot\left(\left(|\nabla u|^{p}+1\right) \nabla u\right) d x \\
= & 2 \int_{\partial \Omega} u_{t}\left(|\nabla u|^{p}+1\right) \frac{\partial u}{\partial \nu} d S \\
& -2 \int_{\Omega} u_{t} \nabla \cdot\left(\left(|\nabla u|^{p}+1\right) \nabla u\right) d x .
\end{aligned}
$$

Substituting (40) into (39), we have

$$
\Phi^{\prime}(t)=2 \int_{\Omega} u_{t}^{2} d x>0
$$

which with $\Phi(0)>0$ imply $\Phi(t)>0$ for all $t \in\left(0, t^{*}\right)$. Making use of the Schwarz inequality, we obtain

$$
2(1+\beta) \Psi^{\prime} \Phi \leq\left(\Psi^{\prime}(t)\right)^{2}=4\left(\int_{\Omega} u u_{t} d x\right)^{2} \leq 2 \Psi(t) \Phi^{\prime}(t) .
$$


Multiplying the above inequality by $\Psi^{-2-\beta}$, we deduce

$$
\left(\Phi \Psi^{-(1+\beta)}\right)^{\prime} \geq 0
$$

Arguing as in Theorem 3.1 in [20], we find

$$
t^{*} \leq T=\frac{1}{2 \beta(1+\beta)}(\Psi(0))^{-\beta}=\frac{\Psi(0)}{2 \beta(1+\beta) \Phi(0)}
$$

valid for $\beta>0$. If $\beta=0$, we have

$$
\Psi(t) \geq \Psi(0) e^{2 M t}
$$

valid for $t>0$, implying that $t^{*}=\infty$. This completes the proof of Theorem 2 .

\section{Lower Bounds for $t^{*}$}

In this section, under the assumption that $\Omega$ is a star shaped domain in $R^{3}$, convex in two orthogonal directions, we seek a lower bound for the blow-up time $t^{*}$. Now we state the result as follows.

Theorem 3. Let $u(x, t)$ be the nonnegative solution of problem (1)-(3) and $u(x, t)$ blows up at $t^{*}$; moreover, the nonnegative functions $f$ and $g$ satisfy

$$
\begin{array}{ll}
f(\xi) \geq k_{1} \xi^{q}, & \xi \geq 0, \\
g(\xi) \leq k_{2} \xi^{s}, & \xi \geq 0,
\end{array}
$$

with $k_{1}>0, k_{2}>0, q>1, s>1, q<s$. Define

$$
\varphi(t)=\int_{\Omega} u^{n(s-1)} d x
$$

where $n$ is a parameter restricted by the condition

$$
n>\max \left\{4, \frac{2}{s-1}\right\} .
$$

Then $\varphi(t)$ satisfies inequality

$$
\varphi^{\prime}(t) \leq \Gamma(\varphi)
$$

for some computable function $\Gamma(\varphi)$. It follows that ${ }^{*}$ is bounded from below. We have

$$
t^{*} \geq \int_{\varphi(0)}^{\infty} \frac{d \eta}{\Gamma(\eta)} d \eta
$$

Proof. Differentiating (47) and making use of the boundary condition (2) together with the conditions (46), we have

$$
\begin{aligned}
& \varphi^{\prime}(t)=n(s-1) \int_{\Omega} u^{n(s-1)-1} u_{t} d x \\
& =n(s-1) \int_{\Omega} u^{n(s-1)-1} \\
& \times\left[\left(\left(|\nabla u|^{p}+1\right) u_{, i}\right)_{, i}-f(u)\right] d x \\
& =n(s-1) \int_{\partial \Omega} u^{n(s-1)-1}\left(|\nabla u|^{p}+1\right) \frac{\partial u}{\partial \nu} d S \\
& -n(s-1)[n(s-1)-1] \\
& \times \int_{\Omega} u^{n(s-1)-2}|\nabla u|^{p+2} d x \\
& -n(s-1)[n(s-1)-1] \\
& \times \int_{\Omega} u^{n(s-1)-2}|\nabla u|^{2} d x \\
& -n(s-1) \int_{\Omega} u^{n(s-1)-1} f(u) d x \\
& \leq k_{2} n(s-1) \int_{\partial \Omega} u^{(n+1)(s-1)} d S \\
& -n(s-1)[n(s-1)-1] \\
& \times \int_{\Omega} u^{n(s-1)-2}|\nabla u|^{p+2} d x \\
& -n(s-1)[n(s-1)-1] \\
& \times \int_{\Omega} u^{n(s-1)-2}|\nabla u|^{2} d x \\
& -k_{1} n(s-1) \int_{\Omega} u^{n(s-1)+q-1} d x .
\end{aligned}
$$

Applying inequality (2.7) in [20] to the first term on the right hand side of (51), we have

$$
\begin{aligned}
\int_{\partial \Omega} u^{(n+1)(s-1)} d S \leq & \frac{3}{\rho_{0}} \int_{\Omega} u^{(n+1)(s-1)} d x \\
& +\frac{(n+1)(s-1) d}{\rho_{0}} \int_{\Omega} u^{(n+1)(s-1)-1}|\nabla u| d x .
\end{aligned}
$$


Substituting (52) into (51), we obtain

$$
\begin{aligned}
\varphi^{\prime}(t) \leq & \frac{3 k_{2} n(s-1)}{\rho_{0}} \int_{\Omega} u^{(n+1)(s-1)} d x \\
& +\frac{k_{2} n(n+1)(s-1)^{2} d}{\rho_{0}} \int_{\Omega} u^{(n+1)(s-1)-1}|\nabla u| d x \\
& -n(s-1)[n(s-1)-1] \int_{\Omega} u^{n(s-1)-2}|\nabla u|^{p+2} d x \\
& -n(s-1)[n(s-1)-1] \int_{\Omega} u^{n(s-1)-2}|\nabla u|^{2} d x \\
& -k_{1} n(s-1) \int_{\Omega} u^{n(s-1)+q-1} d x .
\end{aligned}
$$

Making use of arithmetic-geometric mean inequality, we derive

$$
\begin{aligned}
\int_{\Omega} u^{(n+1)(s-1)-1}|\nabla u| d x \leq & \frac{\mu}{2} \int_{\Omega} u^{n(s-1)-2}|\nabla u|^{2} d x \\
& +\frac{1}{2 \mu} \int_{\Omega} u^{(n+2)(s-1)} d x
\end{aligned}
$$

for all $\mu>0$. Choose $\mu>0$ such that

$$
\frac{k_{2} n(n+1)(s-1)^{2} d \mu}{2 \rho_{0}}-n(s-1)[n(s-1)-1]=0
$$

We rewrite (53) as

$$
\begin{aligned}
\varphi^{\prime}(t) \leq & \frac{3 k_{2} n(s-1)}{\rho_{0}} \int_{\Omega} u^{(n+1)(s-1)} d x \\
& +\frac{k_{2} n(n+1)(s-1)^{2} d}{2 \mu \rho_{0}} \int_{\Omega} u^{(n+2)(s-1)} d x \\
& -n(s-1)[n(s-1)-1] \int_{\Omega} u^{n(s-1)-2}|\nabla u|^{p+2} d x \\
& -k_{1} n(s-1) \int_{\Omega} u^{n(s-1)+q-1} d x .
\end{aligned}
$$

Using Hölder inequality, we get

$$
\begin{aligned}
\int_{\Omega} u^{n(s-1)} d x \leq & \left(\int_{\Omega} u^{n(s-1)+q-1} d x\right)^{n(s-1) /(n(s-1)+q-1)} \\
& \times|\Omega|^{(q-1) /(n(s-1)+q-1)}
\end{aligned}
$$

Combining (56) with (57), we obtain

$$
\begin{aligned}
\varphi^{\prime}(t) \leq & \frac{3 k_{2} n(s-1)}{\rho_{0}} \int_{\Omega} u^{(n+1)(s-1)} d x \\
& +\frac{k_{2} n(n+1)(s-1)^{2} d}{2 \mu \rho_{0}} \int_{\Omega} u^{(n+2)(s-1)} d x \\
& -n(s-1)[n(s-1)-1] \\
& \times \int_{\Omega} u^{n(s-1)-2}|\nabla u|^{p+2} d x \\
& -k_{1} n(s-1)|\Omega|^{(1-q) / n(s-1)} \varphi^{(n(s-1)+q-1) / n(s-1)} \\
= & \frac{3 k_{2} n(s-1)}{\rho_{0}} J_{1}(t) \\
& +\frac{k_{2} n(n+1)(s-1)^{2} d}{2 \mu \rho_{0}} J_{2}(t) \\
& -n(s-1)[n(s-1)-1] \omega(t) \\
& -k_{1} n(s-1)|\Omega|^{(1-q) / n(s-1)} \varphi^{(n(s-1)+q-1) / n(s-1)},
\end{aligned}
$$

where

$$
\begin{gathered}
J_{1}(t)=\int_{\Omega} u^{(n+1)(s-1)} d x, \\
J_{2}(t)=\int_{\Omega} u^{(n+2)(s-1)} d x, \\
\omega(t)=\int_{\Omega} u^{n(s-1)-2}|\nabla u|^{p+2} d x .
\end{gathered}
$$

Using Sobolev type inequality (A.5) derived by Payne et al. [21], we obtain

$$
\begin{aligned}
J_{1}(t)= & \int_{\Omega} u^{(n+1)(s-1)} d x \\
\leq & \left\{\frac{3}{\rho_{0}} \int_{\Omega} u^{(2 / 3)(n+1)(s-1)} d x+\frac{(n+1)(s-1)}{3}\right. \\
& \left.\times\left(1+\frac{d}{\rho_{0}}\right) \int_{\Omega} u^{(2 / 3)(n+1)(s-1)-1}|\nabla u| d x\right\}^{3 / 2} .
\end{aligned}
$$

We now make use of Hölder inequality to bound the second integral on the right hand side of (60) as follows:

$$
\begin{aligned}
& \int_{\Omega} u^{(2 / 3)(n+1)(s-1)-1}|\nabla u| d x \\
& \quad \leq\left(\int_{\Omega} u^{(2 / 3)(n+1)(s-1)\left(1-\delta_{1}\right)} d x\right)^{(p+1) /(p+2)} \omega^{1 /(p+2)},
\end{aligned}
$$

with

$$
\delta_{1}=\frac{(n-2)(s-1)+3 p}{2(n+1)(s-1)(p+1)} \text {. }
$$


We note that $\delta_{1}<1$ for $n>(3 p-2(s-1)(p+2)) /(s-1)(2 p+1)$, an inequality satisfied in view of (48). Using again Hölder's inequality, we obtain

$$
\begin{gathered}
\int_{\Omega} u^{(2 / 3)(n+1)(s-1)\left(1-\delta_{1}\right)} d x \\
\leq \varphi^{2(n+1)\left(1-\delta_{1}\right) / 3 n}|\Omega|^{1-\left(2(n+1)\left(1-\delta_{1}\right) / 3 n\right)}, \\
\int_{\Omega} u^{(2 / 3)(n+1)(s-1)} d x \leq \varphi^{2(n+1) / 3 n}|\Omega|^{1-(2(n+1) / 3 n)},
\end{gathered}
$$

where $|\Omega|=\int_{\Omega} d x$ is the volume of $\Omega$. Substituting (61) and (63) in (60), we obtain the following inequality:

$$
\begin{aligned}
J_{1}(t) \leq & \left\{\widetilde{c_{1}} \varphi^{2(n+1) / 3 n}+\widetilde{c_{2}} \varphi^{\left(2(n+1)\left(1-\delta_{1}\right) / 3 n\right)((p+1) /(p+2))}\right. \\
& \left.\times \omega^{1 /(p+2)}\right\}^{3 / 2} \\
\leq & c_{1} \varphi^{(n+1) / n}+c_{2} \varphi^{\left((n+1)\left(1-\delta_{1}\right) / n\right)((p+1) /(p+2))} \omega^{3 / 2(p+2)},
\end{aligned}
$$

where $c_{1}, c_{2}$ are computable positive constants. Note that the last inequality in (64) follows from Hölder inequality under the particular form $(a+b)^{3 / 2} \leq \sqrt{2}\left(a^{3 / 2}+b^{3 / 2}\right)$. Similarly, we can bound $J_{2}$ and get

$$
J_{2}(t) \leq c_{3} \varphi^{(n+2) / n}+c_{4} \varphi^{\left((n+2)\left(1-\delta_{2}\right) / n\right)((p+1) /(p+2))} \omega^{3 / 2(p+2)},
$$

where $c_{3}, c_{4}$ are computable positive constants,

$$
\delta_{2}=\frac{(n-4)(s-1)+3 p}{2(n+1)(s-1)(p+1)} \text {. }
$$

We note that $\delta_{2}<1$ for $n>(3 p-4(s-1)(p+2)) /(s-1)(2 p+1)$, an inequality satisfied in view of (48). Inserting (64) and (65) in (58), we arrive at

$$
\begin{aligned}
\varphi^{\prime}(t) \leq & \widetilde{d_{1}} \varphi^{(n+1) / n}+\widetilde{d_{2}} \varphi^{\left((n+1)\left(1-\delta_{1}\right) / n\right) \lambda} \omega^{3 / 2(p+2)} \\
& +d_{3} \varphi^{(n+2) / n}+\widetilde{d_{4}} \varphi^{\left((n+2)\left(1-\delta_{2}\right) / n\right) \lambda} \omega^{3 / 2(p+2)} \\
& -n(s-1)[n(s-1)-1] \omega(t) \\
& -k_{1} n(s-1)|\Omega|^{(1-q) / n(s-1)} \varphi^{(n(s-1)+q-1) / n(s-1)},
\end{aligned}
$$

where $\lambda=(p+1) /(p+2), d_{3}$ and $\widetilde{d_{j}}(j=1,2,4)$ are computable positive constants. Next, we want to eliminate the quantity $\omega(t)$ in inequality (67). By using the following inequality:

$$
\begin{aligned}
\varphi^{\alpha} \omega^{\beta} & =(\gamma \omega)^{\beta}\left\{\frac{\varphi^{\alpha /(1-\beta)}}{\gamma^{\beta /(1-\beta)}}\right\}^{1-\beta} \\
& \leq \gamma \beta \omega+(1-\beta) \gamma^{\beta /(\beta-1)}+(1-\beta) \gamma^{\beta /(\beta-1)} \varphi^{\alpha /(1-\beta)}
\end{aligned}
$$

valid for $0<\beta<1$, where $\gamma$ is an arbitrary positive constant, then we have

$$
\begin{aligned}
& \widetilde{d}_{2} \varphi^{\left((n+1)\left(1-\delta_{1}\right) / n\right) \lambda} \omega^{3 / 2(p+2)} \\
& \quad \leq \gamma_{1} \omega(t)+d_{2} \varphi^{\left(2(n+1)\left(1-\delta_{1}\right)(p+2) / n(2 p+1)\right) \lambda}, \\
& \widetilde{d_{4}} \varphi^{\left((n+2)\left(1-\delta_{2}\right) / n\right) \lambda} \omega^{3 / 2(p+2)} \\
& \quad \leq \gamma_{2} \omega(t)+d_{4} \varphi^{\left(2(n+2)\left(1-\delta_{2}\right)(p+2) / n(2 p+1)\right) \lambda},
\end{aligned}
$$

with arbitrary positive constants $\gamma_{1}, \gamma_{2}$ and computable positive constants $d_{2}, d_{4}$. Substitute (69) in (67) and choose the arbitrary (positive) constants $\gamma_{1}, \gamma_{2}$ such that $\gamma_{1}+\gamma_{2}-n(s-$ 1) $[n(s-1)-1]=0$. We obtain

$$
\begin{aligned}
\varphi^{\prime}(t) \leq & \widetilde{d_{1}} \varphi^{(n+1) / n}+d_{2} \varphi^{\left(2(n+1)\left(1-\delta_{1}\right)(p+2) / n(2 p+1)\right) \lambda} \\
& +d_{3} \varphi^{(n+2) / n}+d_{4} \varphi^{\left(2(n+2)\left(1-\delta_{2}\right)(p+2) / n(2 p+1)\right) \lambda} \\
& -k_{1} n(s-1)|\Omega|^{(1-q) / n(s-1)} \varphi^{(n(s-1)+q-1) / n(s-1)} .
\end{aligned}
$$

We eliminate the last term in (70), by using the following inequality:

$$
\begin{aligned}
\varphi^{(n+1) / n}= & \left\{m \varphi^{(n(s-1)+q-1) / n(s-1)}\right\}^{(2 n-1)(s-1) /((2 n-1)(s-1)+s-q)} \\
& \times\left\{m^{(2 n-1)(1-s) /(s-q)} \varphi^{3}\right\}^{(s-q) /((2 n-1)(s-1)+s-q)} \\
\leq & \frac{(2 n-1)(s-1)}{(2 n-1)(s-1)+s-q} m \varphi^{(n(s-1)+q-1) / n(s-1)} \\
& +\frac{s-q}{(2 n-1)(s-1)+s-q} m^{(2 n-1)(1-s) /(s-q)} \varphi^{3},
\end{aligned}
$$

valid for $q<s$ and arbitrary $m>0$, and choose $m$ such that

$$
\frac{(2 n-1)(s-1)}{(2 n-1)(s-1)+s-q} \widetilde{d_{1}} m-k_{1} n(s-1)|\Omega|^{(1-q) / n(s-1)}=0 \text {. }
$$

Then (70) can be rewritten as

$$
\begin{aligned}
\varphi^{\prime}(t) \leq & d_{1} \varphi^{3}+d_{2} \varphi^{\left(2(n+1)\left(1-\delta_{1}\right)(p+2) / n(2 p+1)\right) \lambda} \\
& +d_{3} \varphi^{(n+2) / n}+d_{4} \varphi^{\left(2(n+2)\left(1-\delta_{2}\right)(p+2) / n(2 p+1)\right) \lambda} .
\end{aligned}
$$

Integrating $(73)$ over $[0, t]$, we conclude

$$
\begin{aligned}
t^{*} \geq \int_{\varphi(0)}^{\infty} d \eta & \\
& \times\left(d_{1} \eta^{3}+d_{2} \eta^{\left(2(n+1)\left(1-\delta_{1}\right)(p+2) / n(2 p+1)\right) \lambda}\right. \\
& \left.+d_{3} \eta^{(n+2) / n}+d_{4} \eta^{\left(2(n+2)\left(1-\delta_{2}\right)(p+2) / n(2 p+1)\right) \lambda}\right)^{-1} .
\end{aligned}
$$

This completes the proof of Theorem 3. 


\section{Conflict of Interests}

The authors declare that there is no conflict of interests regarding the publication of this paper.

\section{Authors' Contribution}

All authors contributed equally to the paper and read and approved the final paper.

\section{Acknowledgments}

This work is supported by the Natural Science Foundation of Shandong Province of China (ZR2012AM018) and the Fundamental Research Funds for the Central Universities (no. 201362032). The authors would like to deeply thank all the reviewers for their insightful and constructive comments.

\section{References}

[1] J. Bebernes and D. Eberly, Mathematical Problems from Combustion Theory, vol. 83 of Applied Mathematical Sciences, Springer, New York, NY, USA, 1989.

[2] C. V. Pao, Nonlinear Parabolic and Elliptic Equations, Plenum Press, New York, NY, USA, 1992.

[3] J. L. Vazquez, The Porous Medium Equations: Mathematical Theory, Oxford University Press, Oxford, UK, 2007.

[4] J. Filo, "Diffusivity versus absorption through the boundary," Journal of Differential Equations, vol. 99, no. 2, pp. 281-305, 1992.

[5] H. A. Levine and L. E. Payne, "Nonexistence theorems for the heat equation with nonlinear boundary conditions and for the porous medium equation backward in time," Journal of Differential Equations, vol. 16, pp. 319-334, 1974.

[6] B. Straughan, Explosive Instabilities in Mechanics, Springer, Berlin, Germany, 1998.

[7] A. A. Samarskii, V. A. Galaktionov, S. P. Kurdyumov, and A. P. Mikhailov, Blow-Up in Quasilinear Parabolic Equations, vol. 19 of de Gruyter Expositions in Mathematics, Walter de Gruyter, Berlin, Germany, 1995.

[8] P. Quittner and P. Souplet, Superlinear Parabolic Problems: BlowUp, Global Existence and Steady States, Birkhäuser Advanced Texts, Birkhäuser, Basel, Switzerland, 2007.

[9] C. Bandle and H. Brunner, "Blowup in diffusion equations: a survey," Journal of Computational and Applied Mathematics, vol. 97, no. 1-2, pp. 3-22, 1998.

[10] V. A. Galaktionov and J. L. Vázquez, "The problem of blowup in nonlinear parabolic equations," Discrete and Continuous Dynamical Systems, vol. 8, no. 2, pp. 399-433, 2002.

[11] H. A. Levine, "The role of critical exponents in blowup theorems," SIAM Review, vol. 32, no. 2, pp. 262-288, 1990.

[12] H. A. Levine, "Nonexistence of global weak solutions to some properly and improperly posed problems of mathematical physics: the method of unbounded Fourier coefficients," Mathematische Annalen, vol. 214, pp. 205-220, 1975.

[13] L. E. Payne, G. A. Philippin, and P. W. Schaefer, "Blow-up phenomena for some nonlinear parabolic problems," Nonlinear Analysis: Theory, Methods \& Applications, vol. 69, no. 10, pp. 3495-3502, 2008.

[14] M. Marras and S. Vernier Piro, "On global existence and bounds for blow-up time in nonlinear parabolic problems with time dependent coefficients," Discrete and Continuous Dynamical Systems, vol. 2013, pp. 535-544, 2013.

[15] Y. Li, Y. Liu, and C. Lin, "Blow-up phenomena for some nonlinear parabolic problems under mixed boundary conditions," Nonlinear Analysis: Real World Applications, vol. 11, no. 5, pp. 3815-3823, 2010.

[16] Y. Li, Y. Liu, and S. Xiao, "Blow-up phenomena for some nonlinear parabolic problems under Robin boundary conditions," Mathematical and Computer Modelling, vol. 54, no. 11-12, pp. 3065-3069, 2011.

[17] C. Enache, "Blow-up phenomena for a class of quasilinear parabolic problems under Robin boundary condition," Applied Mathematics Letters, vol. 24, no. 3, pp. 288-292, 2011.

[18] J. Ding, "Global and blow-up solutions for nonlinear parabolic equations with Robin boundary conditions," Computers \& Mathematics with Applications, vol. 65, no. 11, pp. 1808-1822, 2013.

[19] Y. Liu, "Blow-up phenomena for the nonlinear nonlocal porous medium equation under Robin boundary condition," Computers \& Mathematics with Applications, vol. 66, no. 10, pp. 2092 2095, 2013.

[20] L. E. Payne, G. A. Philippin, and S. Vernier Piro, "Blowup phenomena for a semilinear heat equation with nonlinear boundary conditon, I," Zeitschrift für Angewandte Mathematik und Physik, vol. 61, no. 6, pp. 999-1007, 2010.

[21] L. E. Payne, G. A. Philippin, and S. Vernier Piro, "Blowup phenomena for a semilinear heat equation with nonlinear boundary condition, II," Nonlinear Analysis: Theory, Methods \& Applications, vol. 73, no. 4, pp. 971-978, 2010.

[22] Y. Liu, "Lower bounds for the blow-up time in a non-local reaction diffusion problem under nonlinear boundary conditions," Mathematical and Computer Modelling, vol. 57, no. 3-4, pp. 926931, 2013.

[23] Y. Liu, S. Luo, and Y. Ye, "Blow-up phenomena for a parabolic problem with a gradient nonlinearity under nonlinear boundary conditions," Computers \& Mathematics with Applications, vol. 65, no. 8, pp. 1194-1199, 2013.

[24] Z. B. Fang, R. Yang, and Y. Chai, "Lower bounds estimate for the blow-up time of a slow diffusion equation with nonlocal source and inner absorption," Mathematical Problems in Engineering, vol. 2014, Article ID 764248, 6 pages, 2014. 


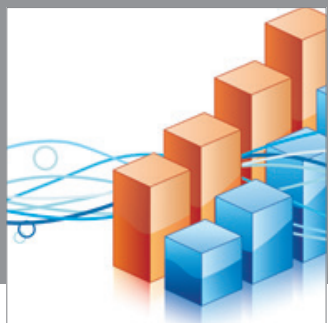

Advances in

Operations Research

mansans

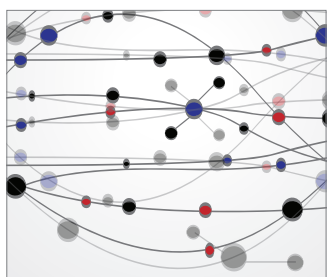

The Scientific World Journal
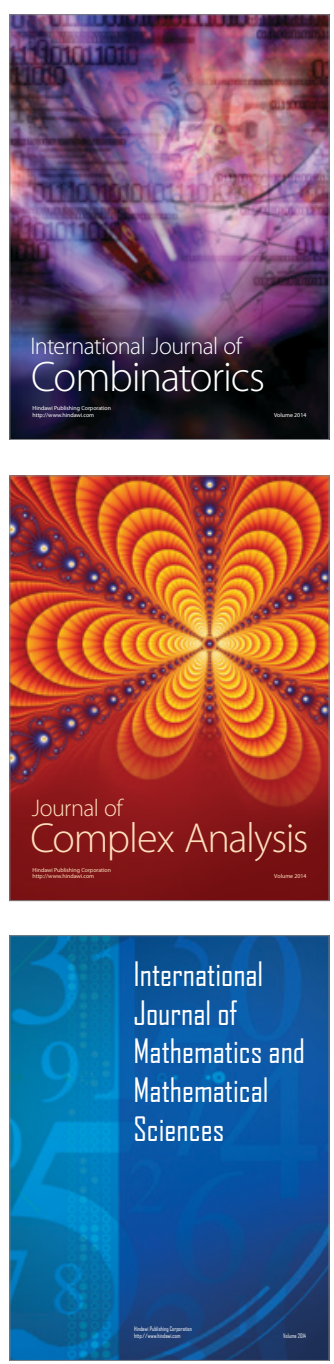
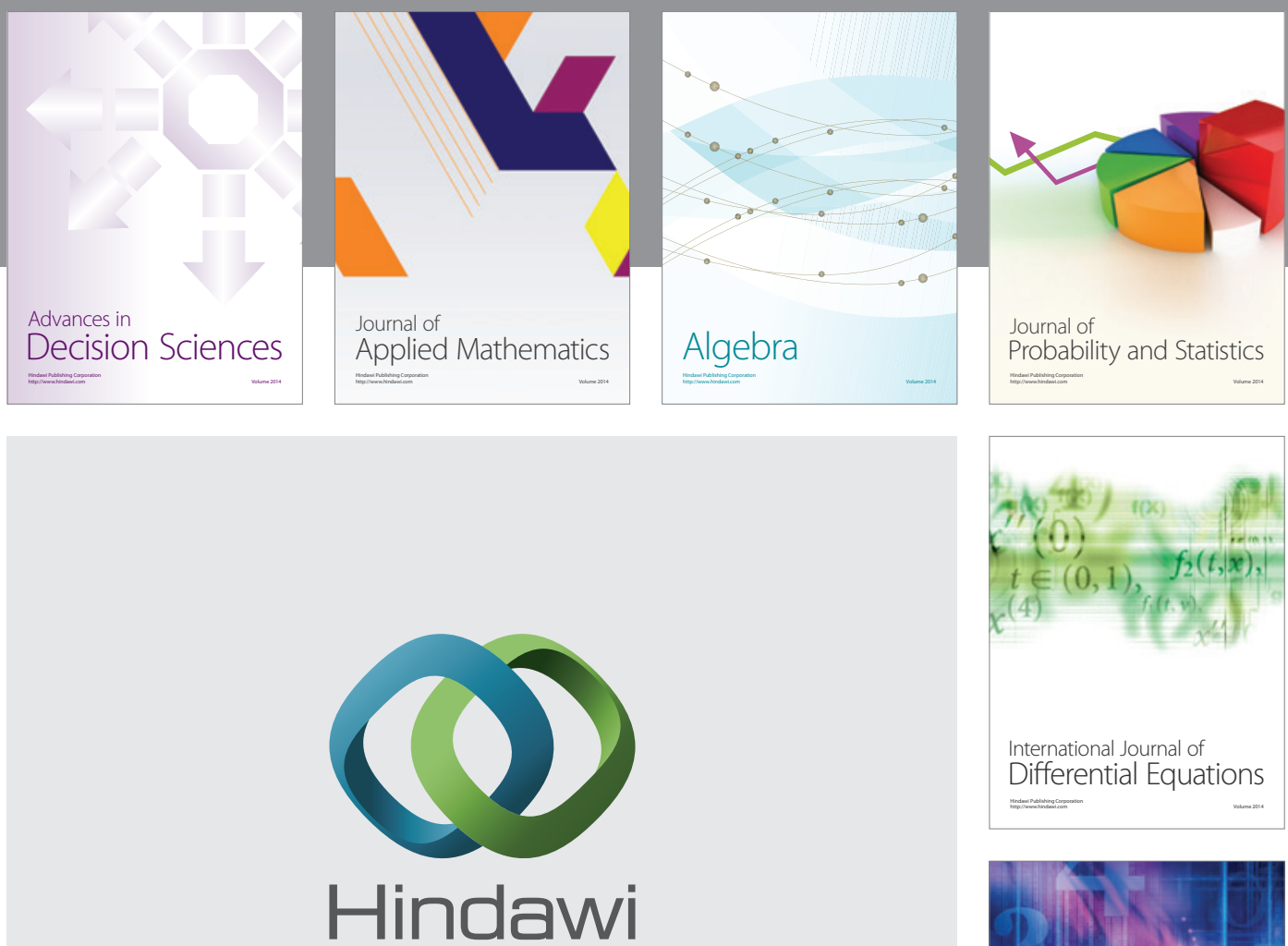

Submit your manuscripts at http://www.hindawi.com
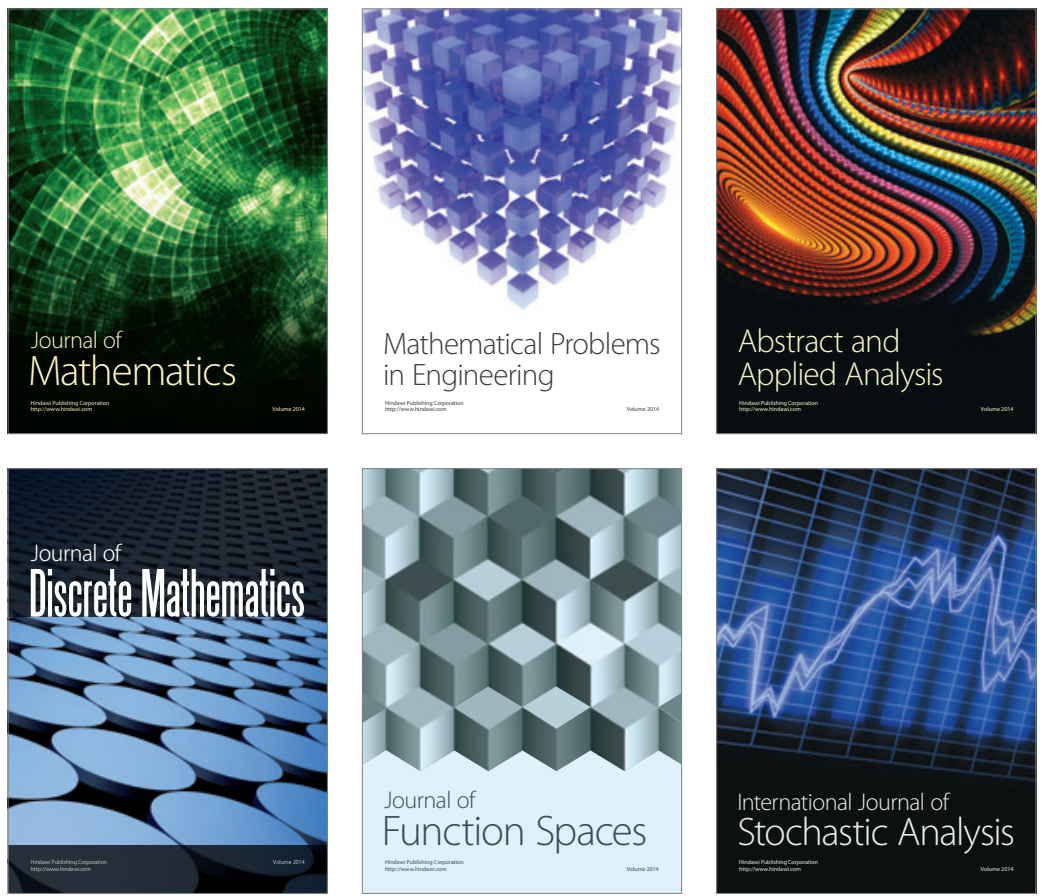

Journal of

Function Spaces

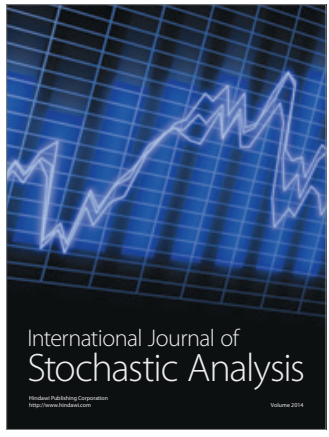

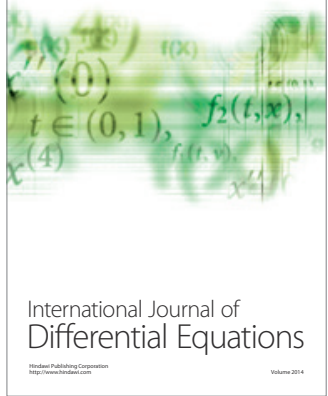
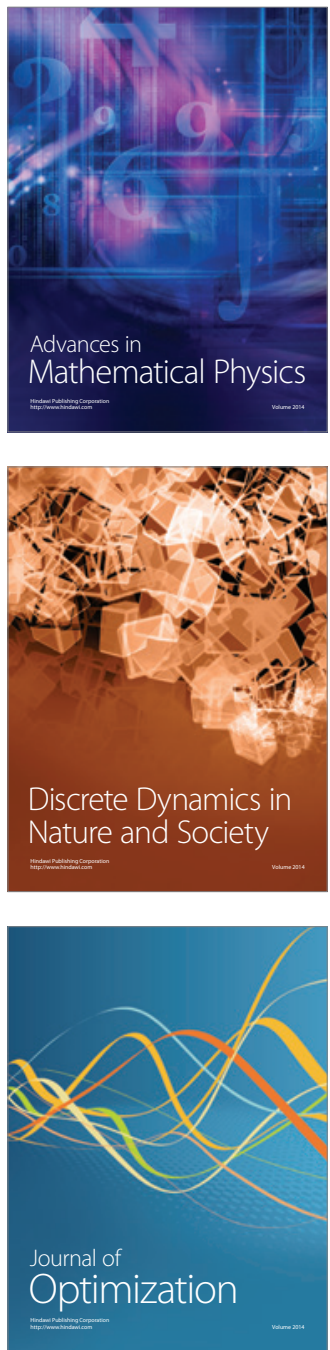